\section{THU0619 PREVALENCE OF PNEUMOCOCCAL VACCINATION IN RHEUMATOLOGIC PATIENTS WITH COMMUNITY ACQUIRED PNEUMONIA. BIOBADASAR REGISTRY}

G. Gómez, A. Brigante, A. Benitez, O. Cerda, S. Retamozo, I. Gandino, A. Quinteros, I. Exeni, B. Barrios, P. Astesana, C. Sanchez Andia, M.V. Collado, A. Granel, A.M. Cappuccio, R. Quintana, E. Mussano, A. Smichowski, M. de la Sota, K. Kirmayr, E. Velozo, M.S. Larroude, A. Bertoli, S. Agüero, C. Battagliotti, S. Soaures de Souza, E. Cavillon, A. Bohr, O. Rillo, L. Carlevaris, E. Bedoya, E. Kerzberg, B. Kisluk, I. Petkovic, D. Pereira, J.C. Barreira, L. Somma, C. Costi, F. Melo, B. Virasoro, S. Paira, L. Roa Perez, G. Casado, D.L.V. María Celina. Sociedad Argentina de Reumatologia, Buenos Aires, Argentina

Background: Biobadasar is a registry that monitors adverse events in patients who use biological treatments in rheumatologic diseases conducted by the Argentine Society of Rheumatology. As in others international registries the community acquired pneumonia (CAP) has been detected as one of the most frequent infectological adverse events. Although all immunosuppressed patients should be vaccinated against streptococcus pneumoniae, there is a proportion of patients who are not.

Objectives: Evaluate the prevalence of pneumococcal vaccination in patients with CAP within the Biobadasar database. Assess factors associated with Severe CAP in these patients.

Methods: A cross-sectional, multicentric study was made in BIOBADASAR database from 2010 to2016.

In patients who reported CAP data of demographics, comorbidities and state of pneumococcal immunization was collected. Microbiological data, treatment and outcome of the event were considered. The severity of CAP was assessed according to the opinion of the attending physician, hospitalization, risk of life and/or death. Values are expressed as mean \pm standard deviation, median (ranges) and frequencies (percentages), as appropriate. We performed bivariate and multivariate logistic regression analysis to identify variables associated with the event.

Results: Of the 4029 patients enrolled in the registry, the cumulative incidence of CAP was $4.2 \%$ ( $n$ 170), $72.4 \%$ ( $n$ 123) were women. The mean age was 57 (SD +/- 14.5). Biological treatment was found in $81.8 \%$ (n 139). Patient $\mathrm{s}$ that have received the pneumococcal vaccine were $40.6 \%(n=69)$. Severe CAP was detected in $7.1 \%$. Streptococco Pneumoniae was the main pathogen isolated in $13 \%$ of the cases. Overall mortality was $4.1 \%$. In the univariate analysis for severe CAP we found statistical significance for Smoking OR 3.88, Cl95 1.063-14.22, $p=$ 0.029 and chronic kidney disease (CKD) OR 31, Cl95 2.6-376, $p=0.007$. When performing a multiple logistic regression model, only renal failure OR $7.39 \mathrm{Cl} 195$ $0.003-0.38 p=0.007$ was a predictor of severe CAP. Not significative association with immunosuppressive treatment ( $p: 0.09)$, age ( $p: 0.464)$, or vaccination ( $p$ : 0.937)

Conclusions: The annual incidence of CAP in Argentina varies between 0.5 $1.1 \%$ while in our cohort it was four times higher. The prevalence of pneumococcal vaccination was less than $50 \%$, showing that, although the literature and guidelines establish the need for vaccination, this is not so in the real world. In the multivariate analysis, only CKD was related to severe CAP. Although in the univariate analysis the CKD and the smoking habit represented factors associated with severity. We must emphasize the medical education in following the international vaccination guidelines.

Disclosure of Interest: None declared

DOI: 10.1136/annrheumdis-2017-eular.5282

\section{THU0620 PERSISTENCE WITH BIOLOGICAL DISEASE-MODIFYING ANTIRHEUMATIC DRUGS - A RETROSPECTIVE DATABASE STUDY IN JAPANESE PATIENTS WITH RHEUMATOID ARTHRITIS}

J. Mahlich ${ }^{1}$, H. Kameda ${ }^{2}$, R. Sruamsiri ${ }^{1} .{ }^{1}$ Health Economics, Janssen Pharmaceutical KK, Tokyo, Japan; ${ }^{2}$ Toho University Ohashi Medical Center, Tokyo, Japan

Background: Persistence rate is one of the key benefits of biological diseasemodifying antirheumatic drugs (bDMARDs) in the treatment of rheumatoid arthritis (RA). Limited evidence of persistence has been found in Japan and the results are not transferrable across countries and cultures (1-3). In addition, the impact of persistence on healthcare cost is still questionable.

Objectives: To assess persistence rates of bDMARDs for the treatment of RA in Japan and compared resource utilization and treatment costs between persistence and non-persistence groups.

Methods: Data was extracted from a Japanese claims database that included 4,400,000 patients between 2009 and 2015. RA patients who initiated bDMARD treatment (bDMARD-naïve patients) were identified and included in the final analysis. Survival analysis was used to estimated 6-, 12-, and 18- month persistence rates for current bDMARDs. Propensity score matching was applied to control for potential treatment selection bias. Resource utilization and health care costs of treatments were calculated 12 months before and after initiation of bDMARDs treatment and compared between persistence and non-persistence groups.

Results: A total of 6,153 bDMARD-naïve patients were included in the final analysis. The overall 1 -year persistence rate was $85 \%$ (95\% Cl, 84-86).
Persistence of patients treated with golimumab was higher [92\% (95\% Cl, 89-94)] than that with other bDMARDs. Overall, 1-year outpatient visits increased from 10 at baseline to 16 after bDMARD treatment, while the number of hospital admissions declined from 3.3 to 1.6 . Hospital days decreased from 17 at baseline to 12 following treatment. The non-persistence group had a larger increase in outpatient visits after bDMARD initiation compared with the persistence group (8 to 16 vs. 10 to 16 , respectively) and a smaller decrease in hospital admissions (3.1 to 1.9 vs. 3.5 to 1.4 , respectively). Compared to non-persistence group, persistence was associated with a reduction in total healthcare costs of 760 USD. Conclusions: Japanese bDMARD-naïve patients with RA have a high persistence rate of bDMARDs. The reduction in medication costs in non-persistent patients is offset by higher hospitalization costs, making non-persistence more expensive.

References:

[1] Dalén J et al. Rheumatol Int 2016;36(7):987-95

[2] Tang B et al. Clin Ther 2008;30(7):1375-84.

[3] Meissner B et al. J Med Econ 2014;17(4):259-65.

Disclosure of Interest: J. Mahlich Employee of: Janssen Pharmaceutical KK, H. Kameda Grant/research support from: Abbvie GK, Astellas Pharma, Chugai Pharmaceutical Co, Ltd., Eisai Co. Ltd., Mitsubishi Tanabe Pharma Co., Nippon Kayaku Co. Ltd., Santen Pharmaceutical Co. Ltd., Takeda Pharmaceutical Co. Ltd, Consultant for: Eli Lilly Japan K.K., Novartis Pharma K.K. and Sanofi K.K., Speakers bureau: AbbVie GK, Astellas Pharma, Bristol- Myers K.K., Chugai Pharmaceutical Co. Ltd., Eisai Co. Ltd., Janssen Pharmaceutical K.K., Mitsubishi Tanabe Pharma Co., Nippon Kayaku Co. Ltd., Pfizer Japan Inc., Santen Pharmaceutical Co. Ltd. and Takeda Pharmaceutical Co. Ltd. and UCB Pharma, R. Sruamsiri Employee of: Janssen Pharmaceutical KK

DOI: 10.1136/annrheumdis-2017-eular.2102

\section{THU0621 COMPARISON OF THE EFFECT OF ANTI-TNF THERAPY ON WORK DISABILITY BETWEEN PATIENTS WITH RHEUMATOID ARTHRITIS, ANKYLOSING SPONDYLITIS AND PSORIATIC ARTHRITIS OVER ONE YEAR- REAL LIFE DATA FROM THE CZECH BIOLOGICS REGISTRY ATTRA}

J. Zavada ${ }^{1}$, L. Szczukova ${ }^{2}$, J. Vencovsky ${ }^{1}$, P. Horák ${ }^{3}$, K. Pavelka ${ }^{1}$ on behalf of collaborators of the ATTRA registry. ${ }^{1}$ Rheumatology, Institute of Rheumatology, Prague; ${ }^{2}$ Institute of Biostatistics and Analyses, Faculty of Medicine, Masaryk University, Brno; ${ }^{3}$ Internal Medicine, Faculty of Medicine and Dentistry, Palacký University, Olomouc, Czech Republic

Objectives: To compare the effect of anti-TNF therapy (a-TNF-th) on Work Disability (WD) between RA, PSA and AS. In the Czech Republic (CZ), a-TNF-th is reimbursed for RA if DAS28 $>5.1$ despite therapy with csDMARDs, for PSA if disease is not "adequately controlled" with csDMARDs, and for AS if BASDAl>4 and CRP/ESR elevated above normal. More than $95 \%$ of anti-TNF treated patients in $\mathrm{CZ}$ are followed up in the ATTRA registry.

Methods: Bionäive patients with RA $(n=1085)$, AS $(n=1126)$ and PSA $(n=351)$ starting a-TNF-th with available baseline data on demography, disease duration and physical function, and on working status at baseline and at 12 months were included in the analysis. Patients older than 60 years, on maternity leave or students were excluded. Work status was self-reported by patients as (A) able to work $=$ [(i) employed, or (ii) unemployed and actively seeking employment], or (B) unable to work = [(iii) on sick leave, or (iv) on disability pension]. Regression analyses were performed to examine the predictors of improvement in WD (change $B \rightarrow A$ coded as $1, B \rightarrow A$ as -1 , no change as 0 ) over 1 year.

\begin{tabular}{lcccc} 
Table 1. Baseline characteristics & & & \\
\hline & RA $(n=1085)$ & AS $(n=1126)$ & PSA $(n=351)$ & $p$-value \\
\hline Female & $836(77.1 \%)$ & $263(23.4 \%)$ & $161(45.9 \%)$ & $<\mathbf{0 . 0 0 1}^{\text {ABC }}$ \\
Disease duration & $8.2 \pm 6.6$ & $7.5 \pm 6.9$ & $8.2 \pm 7.2$ & $\mathbf{0 . 0 0 2}^{\mathrm{A}}$ \\
Age at start of anti-TNF Therapy & $46.6 \pm 9.0$ & $38.2 \pm 8.8$ & $44.1 \pm 9.3$ & $<\mathbf{0 . 0 0 1 ^ { A B C }}$ \\
HAQ & $1.5 \pm 0.6$ & $1.1 \pm 0.5$ & $1.2 \pm 0.6$ & $<\mathbf{0 . 0 0 1}^{\text {ABC }}$ \\
Calendar year of starting anti-TNF & & & & \\
$\quad$ before 2008 & $420(38.7 \%)$ & $306(27.2 \%)$ & $90(25.6 \%)$ & $<\mathbf{0 . 0 0 1}^{\text {AB }}$ \\
2009-2012 & $394(36.3 \%)$ & $427(37.9 \%)$ & $156(44.4 \%)$ & \\
2013-2015 & $271(25.0 \%)$ & $393(34.9 \%)$ & $105(29.9 \%)$ & \\
\hline
\end{tabular}

Post-hoc analysis (with Bonferroni correction): statistically significant difference btw groups A) RA vs. AS, B) RA vs. PSA, C) AS vs. PSA. Values or $\mathrm{N}(\%)$ or mean (SD).

Table 2. Prediction of improvement in work disability

\begin{tabular}{|c|c|c|c|c|}
\hline \multirow[t]{2}{*}{ Independent variable } & \multicolumn{2}{|c|}{ Univariate analysis } & \multicolumn{2}{|c|}{ Multivariate analysis } \\
\hline & OR $(95 \% \mathrm{Cl})$ & $\overline{p \text {-value }}$ & OR $(95 \% \mathrm{Cl})$ & $\mathrm{p}$-value \\
\hline \multicolumn{5}{|l|}{ Diagnosis } \\
\hline RA & reference & & reference & \\
\hline AS & $1.477(1.150 ; 1.898)$ & 0.002 & $1.466(1.044 ; 2.058)$ & 0.027 \\
\hline PSA & $1.206(0.840 ; 1.731)$ & 0.310 & $1.226(0.838 ; 1.792)$ & 0.293 \\
\hline Female & $0.821(0.651 ; 1.034)$ & 0.093 & $0.988(0.754 ; 1.295)$ & 0.932 \\
\hline Age at start of anti-TNF therapy & $0.983(0.971 ; 0.994)$ & 0.004 & $0.987(0.974 ; 1.001)$ & 0.067 \\
\hline Disease duration & $0.992(0.975 ; 1.009)$ & 0.341 & $0.998(0.980 ; 1.016)$ & 0.840 \\
\hline $\mathrm{HAQ}$ & $1.024(0.839 ; 1.251)$ & 0.815 & $1.181(0.949 ; 1.470)$ & 0.135 \\
\hline \multicolumn{5}{|l|}{ Calendar year of starting anti-TNF } \\
\hline before 2008 & reference & & reference & \\
\hline 2009-2012 & $0.843(0.639 ; 1.113)$ & 0.228 & $0.799(0.601 ; 1.062)$ & 0.122 \\
\hline 2013-2015 & $1.075(0.804 ; 1.438)$ & 0.624 & $1.001(0.741 ; 1.352)$ & 0.995 \\
\hline
\end{tabular}


Results: Baseline characteristic were significantly different between diagnoses (Table 1). In patients with RA, 60\% were able to work at baseline and $61 \%$ at 12 month, with PSA $71 \%$ and $74 \%$ resp., and with AS $72 \%$ and $77 \%$ resp. The main driver of improvement of WD was a change in the sick leave (RA $6 \% \rightarrow 2 \%$, PSA $7 \% \rightarrow 1 \%$, AS $9 \% \rightarrow 2 \%$ ). In univariate analyses, diagnosis of AS and younger age at start of a-TNF-th were predictive of improvement in WD. In multivariate analysis, only diagnosis of AS was significantly associated with improvement in WD.

Conclusions: These real life data from CZ show that for improvement of WD, a-TNF-th was most effective in patients with AS. This may be a sequel of disease specific factors or reimbursement policy.

Disclosure of Interest: None declared

DOI: 10.1136/annrheumdis-2017-eular.4056

\section{THU0622 PATIENT-CENTERED AGING BIOBANKS - A SURVEY ON PUBLIC PERCEPTIONS AND PATIENT CHOICE AMONG RHEUMATOLOGY OUTPATIENTS}

J.V. Cordeiro ${ }^{1}$, A. Fernandes ${ }^{2}$, C.D. Lopes $^{3}$, G. Victorino ${ }^{4}$, P. Lobato Faria ${ }^{1}$, M.J. Cercas ${ }^{4}$, J.C. Branco ${ }^{5}$, F.M. Pimentel-Santos ${ }^{3,5} .{ }^{1}$ Centro de Investigação em Saúde Pública (CISP), Escola Nacional de Saúde Pública; ${ }^{2}$ CICS.NOVA Centro Interdisciplinar de Ciências Sociais, Faculdade de Ciências Sociais e Humanas, NOVA University of Lisbon; ${ }^{3}$ Rheumatology, CHLO, Hospital Egas Moniz; ${ }^{4}$ NOVA Information Management School (NOVAIMS); ${ }^{5}$ CEDOC, NOVA Medical School/Faculdade Ciências Médicas, NOVA University of Lisbon, Lisboa, Portugal

Background: Biobanks for research (BBR) are organized repositories of biological materials and associated health information with enormous potential and value for scientific research. In consonance with increasing attention to healthy aging research, BBR specifically oriented to chronic diseases and aging populations have gathered heightened attention. Public perceptions and patient choices are key to design, develop and implement patient-centered BBR. Public awareness, education and involvement are confidence building and unequivocally lead to higher participation in scientific enterprises.

Objectives: To assess patient awareness, perception and choices regarding aging biobanking activities.

Methods: We developed and applied a standard anonymous questionnaire to rheumatology terciary outpatients, aged 50 or older, between March-October 2016. Demographic data and perceptions about biobanking were collected. Data analysis was performed using Stata $14^{\circledR}$ software.

Results: We obtained a total of 131 valid responses [age (min-max, 50-93), mean (64); sex ratio (M/F) (40/91, 44\%), education years (min-max, 0-20), mean (8.5)]. $69 \%$ of respondents did not know the specific term "biobank" but $57 \%$ were aware about the possibility of donating their biological material for research purposes. Furthermore, $77 \%$ of respondents indicated they were willing to contribute with their biological material to BBR, stating they had no particular preference whether these infrastructures were of private or public nature. However, they expressed a clear preference for these to be based at scientific research institutes (50\%), instead of hospitals (23\%), universities (16\%) or biotechnology companies $(7 \%)$. Moreover, respondents highlighted diferent requirements for their participation with anonymity $(31 \%)$ and confidentiality $(27 \%)$ ranking as top priorities. Most importantly, a majority of respondents $(70 \%)$ expressed their agreement with a biobank exclusively dedicated to the study of aging, considering that people of older ages have higher disease burdens and that such research infrastructures and practices expressed respect for the particular problems of the elderly (Figure).

Why dedicated Aging Biobanks?

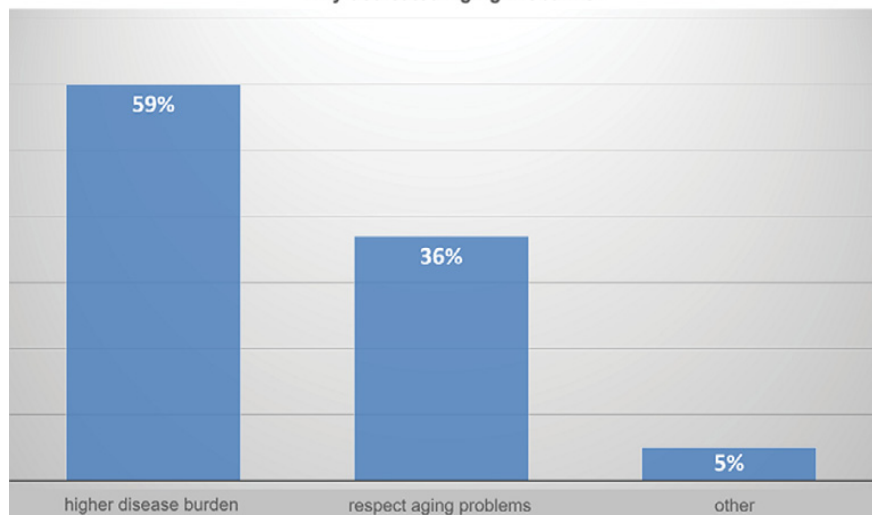

Conclusions: Our study constitutes a comprehensive assessment of public perceptions and patient choices regarding biobanks for aging research purposes among rheumatology outpatients. Although awareness is still suboptimal, BBR are highly regarded health infrastructures with enormous potential for further patient-centered development.

Disclosure of Interest: None declared

DOI: 10.1136/annrheumdis-2017-eular.6527

\section{THU0623 DECREASE IN THE TEMPORARY WORK INCAPACITY DUE TO MUSCULOSKELETIC DISEASES: UTILITY OF A PREVENTIVE PROGRAM IN VALENCIA-LA FE HEALTH AREA}

J.E. Oller Rodríguez ${ }^{1}$, M.T. Fuente Goig ${ }^{2}$, F. Ortiz Sanjuan ${ }^{1}$, J. Ivorra Cortés ${ }^{1}$, E. Grau García ${ }^{1}$, E. Vicens Bernabéu ${ }^{1}$, E. Labrador Sánchez ${ }^{1}$, K.R. Arévalo Ruales $^{1}$, J. Fragio Gil ${ }^{1}$, R. González Mazario ${ }^{1}$, C.P. Alcañiz Escandell ${ }^{1}$, I. Cánovas Olmos ${ }^{1}$, I. Chalmeta Verdejo ${ }^{1}$, C.M. Feced Olmos ${ }^{1}$, L. González Puig ${ }^{1}$, I. Martínez Cordellat ${ }^{1}$, C. Nájera Herranz ${ }^{1}$, R. Negueroles Albuixech ${ }^{1}$, J. Andani Cervera ${ }^{2}$, J.A. Román Ivorra ${ }^{1}$ on behalf of Rheumatology Service. ${ }^{1}$ Rheumatology; ${ }^{2}$ Service of Prevention of Occupational Hazards, Hospital Universitari I Politècnic la Fe, Valencia, Spain

Background: Musculoskeletal diseases are nowadays a frequent cause of temporary work incapacity (TWI). The implementation of specific programs for the care of these patients can be an important cornerstone in the resolution of these TWI processes.

Objectives: Our aim is to analyze the usefulness of an Early Intervention Program $(E I P)$ in patients with TWI of musculoskeletal origin.

Methods: Case-control study, including patients from HUP La Fe area, which were referred from Primary Care since April 2012 to April 2016 to our Early intervention program (EIP) addressed to patients with TWI of musculoskeletal origin. The patients were evaluated in our consultation within a maximum of 15 days after the start of TWI. The intervention consisted in an evaluation, including complementary tests if necessary, diagnosis and treatment by the rheumatologist in consultation. The patient was followed in consultation continuously until discharge. Patients whose disabilities were of traumatic or surgical origin were excluded. A comparative study was carried out against a control group of patients with ILT of musculoskeletal origin.

Results: A total of 666 patients were recruited; 508 (76.3\%) belonged to EIP group (46.1\% male; $53.9 \%$ female) and 158 patients $(23.7 \%)$ belonged to the control group ( $44.3 \%$ male; $55.7 \%$ female).

The average age was $47,6 \pm 10.5$ years for the EIP ones and $46.7 \pm 10$ years in the control group.

The most frequent diagnoses were low back pain $(23.3 \%)$, neck pain $(18 \%)$ y lumbosciatica (12.3\%). In EIP group, $100 \%$ of the patients received medical treatment, a $54.5 \%$ received instructions for doings physical therapy at home, an ultrasound scan was performed to the $26,4 \%$ of the patients while the $19.9 \%$ received at least one local infiltration. The longest TWI corresponded to knee meniscopathy (203 days), painful shoulder syndrome (173 days) and lumbosciatica (170.5 days). No statistically significant differences were found between the duration of the TWI attending to sex, age group, labour activity or diagnose. However, a significant association was found between TWI duration and the delay since the start of the symptons to the referral from Primary Care to our consultation, specially within the firdt 10 days $(p=0.04)$. Furthermore, TWI duration was significantly shorter in the EIP group patients than in the control group patients $(137.4 \pm 132.3$ days vs $194.7 \pm 143.1$ days; $p<0,001)$. The period before relapse was longer in the EIP group, although the differences did not reach significance. In addition, an inverse relation was found between age and time to relapse $(p=0,01)$

Conclusions: The establishment of an early intervention program specifically addressed to patients with temporary work incapacity of musculoskeletal origin shortens the duration of this situation, allowing the patient to rejoin his work activity, with the resulting cost savings related to work incapacity.

Disclosure of Interest: None declared

DOI: 10.1136/annrheumdis-2017-eular.5486

\section{THU0624 ASESSMENT OF PSYCHIC EXPERIENCES IN PATIENTS WITH RHEUMATIC DISEASES}

J.L. Prados-Ojeda ${ }^{1}$, J.L. Garrido-Castro ${ }^{2}$, R. Segura-Ruiz ${ }^{1}$,

C. González-Navas ${ }^{2}$, P. Font-Ugalde ${ }^{2}$, E. Collantes-Estevez ${ }^{2}$,

A. Escudero-Contreras ${ }^{1}$. ${ }^{1}$ Reina Sofia University Hospital; ${ }^{2}$ Maimonides Institute for Biomedical Research of Cordoba, Cordoba, Spain

Background: There are several studies that report psychiatric comorbidity in patients with rheumatic disease, mainly the presence of mood disorders. Some of them describe non-affective psychosis related with inflammatory processes. Population with chronic inflammatory disease could present subclinical psychotic experiences that can interfere in the patient's functional status. The Community Assessment of Psychic Experiences (CAPE) scale is a validated and widely used tool for the evaluation of these experiences in the general population.

Objectives: To identify the presence of psychic experiences in different populations with a diagnosis of rheumatic disease, and to compare it with a sample of healthy subjects.

Methods: 124 subjects completed surveys including SF-12 and CAPE questionnaires, as well as other demographic and behavioral variables. Among them, 70 had Spondylarthritis (SpA) (age 44.3 \pm 13 years, 62\% female), 23 rheumatoid arthritis (RA) (age $51.2 \pm 13$ years, $82 \%$ female) and the rest were individuals without rheumatic diseases ( $47.6 \pm 12$ years, $58 \%$ female).

Results: Results of the SF-12 test in their mental and physical domains, and the CAPE questionnaire in their dimensions (positive, negative, depressive and total symptoms) are shown in the table, expressed as mean value (SD) and in the graph expressed as density histograms with mean values. Significant statistical 